\title{
Écopoèmes : le parti pris de voir l'urgence
}

Une approche du recueil Con los ojos abiertos de Jorge Riechmann

El terco oficio de lucidez en los ecopoemas: breves apuntes acerca del poemario

Con los ojos abiertos de Jorge Riechmann

Judite Rodrigues

\section{(2) OpenEdition}

1 Journals

\section{Édition électronique}

URL : https://journals.openedition.org/agedor/5910

DOI : 10.4000/agedor.5910

ISSN : 2104-3353

Éditeur

Laboratoire LISAA

\section{Référence électronique}

Judite Rodrigues, «Écopoèmes : le parti pris de voir l'urgence », L'Âge d'or [En ligne], 13 | 2020, mis en ligne le 01 octobre 2021, consulté le 14 octobre 2021. URL : http://journals.openedition.org/agedor/ 5910 ; DOI : https://doi.org/10.4000/agedor.5910

Ce document a été généré automatiquement le 14 octobre 2021.

L'Âge d'or. Images dans le monde ibérique et ibéricoaméricain 


\title{
Écopoèmes : le parti pris de voir l'urgence
}

\author{
Une approche du recueil Con los ojos abiertos de Jorge Riechmann \\ El terco oficio de lucidez en los ecopoemas: breves apuntes acerca del poemario \\ Con los ojos abiertos de Jorge Riechmann
}

\section{Judite Rodrigues}

1 Jorge Riechmann (Madrid, 1962) est professeur de philosophie morale à l'Université Autonome de Madrid, mais il est aussi - et sans doute avant tout - poète, essayiste, traducteur (notamment de la poésie de René Char aux éditions Hiperión et Visor). Ses essais sont majoritairement orientés vers les thèmes de l'écologie politique, les transitions post-capitalistes et l'analyse des espoirs techno-phoriques. Ces mêmes étoffes thématiques sont présentes dans sa poésie. Il est à ce jour l'auteur de près d'une quarantaine de recueils de poèmes.

2 Si l'on voulait marquer d'un signe bachelardien la caractérisation de son écriture poétique, c'est sans doute le feu que l'on convoquerait d'abord. En effet, son œuvre est traversée de flammes fuligineuses, de pluies de cendres. Elle dérange, elle bouscule, elle ébranle un lecteur directement interpellé : "en ti que ahora lees esta frase" (157) ${ }^{1}$. C'est donc une expérience de lecture qui ne laisse pas indemne. La vertu des flammes, c'est le refus du « subissement » - pour employer ici un néologisme forgé par Blanchot - c'est donc aussi une écriture qui invite à la réplique et à la réaction. Un vent de colère traverse ainsi toute son œuvre. Le ton devient même parfois menaçant et querelleur :

Nunca, jamás me vuelva a decir nadie

que el fin justifica los medios

o sintiéndolo mucho no respondo de mis fines

ni de mis medios

ni muchísimo menos de mis actos. (100)

3 Cette colère est celle-là même qui sera le moteur et le terreau pour l'action : "convierte la mala leche en buena savia"2 est une consigne reprise dans le recueil El Corte bajo la piel. Et c'est ainsi souvent la rage au ventre et le dégoût aux lèvres que le poète égrène 
les temps douloureux d'un accablant calendrier: "Pero hoy once de noviembre de mi rabia / a las dieciocho cincuenta y cinco de mi asco" (114). C'est assurément une poésie qui procède d'une raison corporelle. De l'aveu même du poète : "Hay demasiado de mí en estos poemas" (118), mais comment pourrait-il en être autrement dans l'œuvre d'un poète pour qui l'action redevient la sœur du rêve.

Jorge Riechmann est un auteur qui refuse le «hors-sol» et qui fait entrer dans le matériau poétique des sujets comme les déchets nucléaires, les supermarchés, les moutons clonés, les fonds de pension ou les perturbateurs endocriniens. Une poésie "tierrafirmista" ${ }^{3}$ dirait sans doute Nicanor Parra. Il n'hésite pas par exemple à introduire des statistiques. Il évoque en d'autres occasions le circuit mondialisé des fraises d'Aranjuez, la précarité, les modèles agricoles, le tourisme sexuel... C'est une poésie au risque du factuel et du prosaïsme. Les adeptes de la poésie hydroponique passeront ici leur chemin. "Los poetas bajaron del Olimpo"4 avait prophétisé Nicanor Parra. Et c'est bien sous la présence tutélaire de l'antipoète que Jorge Riechmann écrit ses écopoèmes.

Cet article a pour objet d'étude une anthologie qui compile des poèmes provenant de onze recueils écrits sur une période de vingt ans, à l'articulation des $\mathrm{xx}^{\mathrm{e}}$ et $\mathrm{xxI}^{\mathrm{e}}$ siècle : Con los ojos abiertos (ecopoemas 1985-2006). Il s'agit d'un recueil qui s'inscrit dans le champ de l'écopoétique en ce sens qu'il s'empare de la question environnementale et qu'il participe d'un travail d'interrogation des gestes d'exclusion. S'il est un mot-clé en écocritique, c'est sans doute le mot " décentrement ». Cette anthologie nous propose en effet de faire un pas de côté : au geste anthropocentré ou egocentré, la poésie de Jorge Riechmann répond par un biais qui invite à repenser l'échange avec le monde et à comprendre les effondrements en cours ${ }^{5}$.

6 Le choix des mots pour dire l'urgence est affaire complexe et exigeante. En voici un premier de lancé : « effondrement » donc. L'effondrement ce n'est pas le désastre (on a sans doute en tête "cette toute-brûlure où toute l'histoire s'est embrasée ${ }^{6}$ dans L'Écriture du désastre de Blanchot). L'effondrement, ce n'est pas non plus l'apocalypse (nul n'est besoin de faire appel aux sirènes inhibitrices d'une apocalypse soudaine et brutale). L'effondrement ce n'est pas la crise (qui ne serait que de passage et nous dédouanerait ainsi de la penser). L'urgence aujourd'hui ce sont les insoutenables de nos mondes qui mènent à l'effondrement, ceux qu'Yves Citton identifie et classe en cinq catégories: l'insoutenable écologique, l'insupportable psychique, l'inacceptable éthique, l'indéfendable politique et l'intenable médiatique ${ }^{7}$.

7 L'urgence qui nous occupe ici n'est ni plus ni moins que l'« arrêt du monde " tel que nous le connaissons (l'expression est celle de Déborah Danowski et Eduardo Viveiros de (astro $\left.^{8}\right)$. Les scientifiques s'accordent aujourd'hui à parler d'effondrement de la civilisation thermo-industrielle. Sur le sujet de l'imminence, de l'irréversibilité, du franchissement des seuils et de l'hypothèse du " point de basculement », la lecture d'un article de Nature publié en 2012 par une vingtaine de chercheurs est tout aussi éclairante qu'édifiante". L'urgence est donc de bousculer les adeptes du "climatoquiétisme $»^{10}$ (ceux pour qui les choses finissent toujours par s'arranger) ou les partisans du " climato-négationnisme $»^{11}$ (les zélés du " circulez, il n'y a rien à voir »), les "aquoibonistes ${ }^{12}$ (qui pensent que faute de mieux, on se rabat sur ce pire qu'il nous reste) et les "cavapétistes $»^{13}$ (les démissionnaires, ceux qui abdiquent sans combattre). Autant d'étiquettes et d'appellations proposées par Bruno Latour, Pablo Servigne et Raphael Stevens. 
8 L'urgence est une perception du temps, c'est un compte-à-rebours lancé. Elle exige, elle commande. La notion d'urgence fait peser lourdement la menace. Et nous sommes impérieusement requis de penser l'urgence du nouveau régime climatique. La question corollaire ne manquera pas d'apparaître : que faire? Car l'effondrement est inévitable mais aucune correction de trajectoire ne semble envisagée.

9 En prenant comme corpus d'étude l'ouvrage Con los ojos abiertos de Jorge Riechmann, on voudrait ici dégager un premier chantier problématique en interrogeant le geste poétique qui dit l'urgence face à l'effondrement. Comment se déploie la poétique de la catastrophe? Quelles stratégies sont mises en place pour rendre compte de ce nouveau régime climatique? Quels affects entrent en jeu et sont convoqués pour faire prendre conscience de l'urgence ? Quel est le rôle de la poésie pour rendre sensible et « donner [d]es figurations vivaces $»^{14}$ de la dégradation des environnements ? La démonstration se structurera sur celle du battement poétique présent dans le recueil : d'abord voir et rendre sensible (l'urgence de la prise de conscience), et, par après, inventer un demain désiré (l'urgence de proposer des alternatives).

\section{Le poète en empêcheur de regarder en rond... l'entêtement à voir}

10 Le titre de cette anthologie, Con los ojos abiertos, fait le pari de la lucidité. Il est question de se confronter à une réalité que les données scientifiques imposent aujourd'hui de voir. Quand le plus grand nombre semble avoir fait le choix de ne pas voir ou de se mentir, ce livre prône résolument le refus de l'aveuglement. Le titre est comme une puissante déclaration de lucidité. Cette inscription affichée au fronton de l'ouvrage semble avertir : «Que nul n'entre ici s'il n'accepte de se dessiller ». À la fin de la lecture, les yeux dessillés, le lecteur n'acceptera sans doute plus de les refermer...

11 Le champ lexical du regard est ainsi particulièrement présent tout au long du recueil : "La lluvia enseña cómo mirar" (302), "Profecía con los ojos casi abiertos" (303), "Quiero ver todo lo que va a venir" (94). C'est donc « les yeux grands ouverts » que commence la lecture du recueil, les yeux fixés sur là où brûle le monde: "C'est parce que la catastrophe constitue un destin détestable dont nous devons dire que nous n'en voulons pas qu'il faut garder les yeux fixés sur elle, sans jamais la perdre de vue $»^{15}$ rappelle le philosophe Jean-Pierre Dupuy.

12 Mais la lucidité comporte cependant sa part de risque : «La lucidité est la blessure la plus rapprochée du soleil $\aleph^{16}$ écrivait René Char. Elle peut déranger. Elle peut démobiliser. Pourtant, la parole poétique prend le risque du syndrome de Cassandre en disant le pire advenu et le pire à venir. Riechmann semble faire sienne la pensée de Hans Jonas : « La prophétie du malheur est faite pour éviter qu'elle se réalise $»^{17}$.

13 Il y a également un poème qui porte le titre du recueil : "Con los ojos abiertos" (94-95). Cette composition a ceci de particulier qu'elle porte une forte puissance de déflagration des instants de violence. Ce ne sont pas ici les mécanismes de la violence qui sont pensés ou analysés mais la seule saillie furieuse de ces moments de brutalité - les coups, les actes, les éruptions télévisuelles de débordements de violence - qui prend place dans le corps poétique :

Quiero ver todo lo que va a venir

[...] 


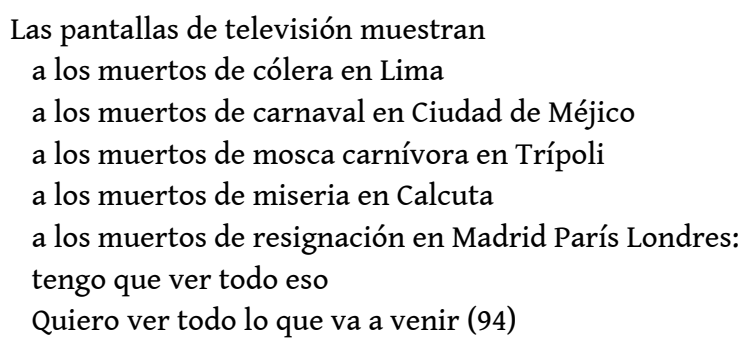

C'est là l'image d'un déchaînement de séquences télévisuelles, une avalanche, un zapping pourrait-on dire, une série qui s'inscrit dans la banalité iconico-médiatique de la violence. Dans cette strophe, la solide, la pénible, charge anaphorique rappelle aussi la fascination que peut exercer l'image de la violence dans nos sociétés contemporaines : une violence sans doute finalement aseptisée car médiatisée et filtrée par un écran, une violence de chaîne d'information en continu qui finit alors par insensibiliser les âmes et anesthésier les mémoires. François Cusset dans son récent essai sur les formes nouvelles de violence parle à cet égard de fascination «magique " et de procédé d'esthétisation : «C'est [...] la fascination magique pour son image [celle de la violence], qui la fige et l'esthétise, l'arrache à tout contexte depuis l'actualité terroriste en continu jusqu'à la surenchère distrayante des jeux et des fictions $»^{18}$.

Voilà un avant-goût de l'écriture par déflagration dans un corps poétique qui fait le choix de montrer les "effractions dans la chair $~^{19}$. C'est donc avec entêtement et obstination que le poète montre que la situation a atteint un degré de gravité inédit. Avec insistance ("Poética de la mosca cojonera », 80), il montre les contradictions et paradoxes en tendant un miroir peu flatteur qui met en lumière les phénomènes de déni. Le poète revêt alors les habits d'une "verde Casandra » (78-79) et en assume la charge de malédiction :

VERDE CASANDRA

Las obviedades

que todo el mundo obstinadamente ignora

las verdades

a las que nadie hace caso

lo requetesabido

pero archirreprimido:

la economía

no puede crecer indefinidamente

dentro de una biosfera finita [...]

L'auteur apparaît ici comme celui qui « voit » et " nomme », néanmoins il endosse aussi le rôle de Cassandre toute de vert vêtue: alertant, annonçant mais sans réussir à convaincre. Les préfixes d'intensification de la première strophe ("requetesabido", "archirreprimido") viennent dire combien les processus sont connus mais aussi combien ils sont devenus familiers et presque banals. Le poème reprend alors dans les strophes suivantes les "vérités fâcheuses et intempestives " ("verdades incómodas"). C'est la voix de l'économiste Serge Latouche dans Le Pari de la décroissance qui semble ainsi ventriloquée: Latouche explique en effet qu'«[...] une croissance infinie est incompatible avec un monde fini $»^{20}$ et Riechmann reprend cette aporie de la croissance illimitée en un monde fini dans la deuxième strophe du poème: "La economía / no puede crecer indefinidamente / dentro de una biosfera finita." La matrice poétique porte ici les voix autorisées, elle se fait l'écho des mises en garde, elle offre son espace 
aux signaux qui disent la déraison de nos mutismes et les impasses de la croissance infinie. L'écho est ici amplifié, répété, prolongé mais semble voué à prendre la forme d'un appel vain et sans portée ("que todo el mundo obstinadamente ignora").

Ce poème vaut pour sa désolation, son impuissance, son désespoir... La question posée est en effet celle de la conséquence nulle, de l'inertie, de l'indifférence, au mieux de l'indolence. La connaissance des faits n'a pas de force dissuasive et transformatrice per se. Faut-il la puissance du spectacle en vraie grandeur pour faire bouger les lignes? C'est la question qui semble s'offrir en conclusion. Mais entre le savoir et l'agir, entre la connaissance et l'action, il y a sans doute un chaînon qui a toute son importance. C'est Jean-Pierre Dupuy qui a rétabli une étape transitionnelle entre ces deux pôles: la croyance. Elle est énoncée dans le paradoxe suivant : « Nous ne croyons pas ce que nous savons $»^{21}$.

\section{Dire et voir l'effondrement : tonalités apocalyptiques}

18 Le mot "crisis" est absent du recueil ${ }^{22}$. Il en est de même pour "catástrofe", "colapso" ou "derrumbe". Le mot "apocalipsis" apparaît lui fugacement, en exergue d'un poème $(35)^{23}$. Cependant le champ lexical de l'effondrement est omniprésent: "exterminio" (89), “arruina" (42), "destruido [...] destruirlo" (42), “agonizan", "mueren” (48) etc. L'arrêt du monde tel que nous le connaissons est ainsi directement nommé : "Perecerá este mundo" (244), "No les quita el sueño el fin del mundo" (152), "El fin del mundo es mañana" (303). Les métaphores de l'effondrement sont nombreuses : "El teatro está en llamas" (49), "la casa se quema" (49), "un borracho de mal vino a quien su carro aplasta" (51), "la escala asesina del confort" (154), "Hacemos como que no pasa / nada, y lo que está pasando / es la demolición del mundo" (54).

19 Dans le premier poème du recueil c'est le mot "desastre" qui est d'abord convoqué ${ }^{24}$ : " [...] la masacre, la pública mentira, la humillación del otro, / la herida en lo común, el desastre ecológico" (29). C'est à un cogito écolo que semble nous inviter ce premier texte : le désastre écologique existe donc- répond le titre du recueil - ouvrons les yeux. Le réel est têtu, il dit sa présence comme un gyrophare, clignotant et hurlant.

20 Un autre de ses poèmes a pour titre "Endzeit" (35). Un titre en allemand qui signifie la fin des temps, le dernier jour, la dernière heure, la fin du règne. C'est le moment du bilan pour dire ce qu'il reste d'une vie. La tonalité est pour le moins désespérée et sans doute si peu révoltée. L'imaginaire est apocalyptique : un survivant plongé dans une atmosphère obscure et ténébreuse. L'idée portée par le poème est celle de l'incapacité, de l'impuissance. La tâche semble vaine et interminable : ce sont des mains de sable qui tentent de retenir le sable. Le début du poème évoque ces mains comme un maillage inopérant ("En vano / intentas retener con tus manos de arena") et les derniers vers complètent la description du supplice ("y en vano intentas / retener la arena / con tus manos rotas"). L'idée du cycle, du retour, de l'interminable est présente dans ces solides répétitions. Et dans ce tourment, comme dans celui de Sisyphe, c'est l'absurdité qui livre sa vérité.

21 À la lecture de ce poème on pense d'abord inévitablement au philosophe Günther Anders et son essai Endzeit und Zeitenende. Gedanken über die atomare Situation ${ }^{25}$ qui était une réflexion sur le nihilisme à l'ère atomique, la menace de l'apocalypse nucléaire et plus généralement sur l'assujettissement à la technique. L'essai consistait aussi en une tentative pour faire prendre conscience des risques d'une réalité nouvelle : ce n'est plus 
l'homme qui est mortel mais bien toute l'humanité. Obsolescente structurellement explique le penseur, cette humanité devient alors «bonne à jeter ${ }^{26}$.

Dans un autre de ses essais, Hiroshima est partout ${ }^{27}$, Anders se parle à lui-même à la deuxième personne, c'est ce même dédoublement qui est mis en œuvre dans ce poème. La deuxième personne est objet d'observation. Ce personnage - tout à la fois soi et un autre - est ici submergé par une vague immense et mortifère, un nuage démesuré et funeste qui envahit l'espace du poème : "Te anega blanco, oceánico, imperial, / el flujo emberrechinado de la muerte". Il est sans doute question de la condition nucléaire, de nos vulnérabilités, de l'anéantissement des vies. Le poète est alors ici «semeur[s] de panique $\aleph^{28}$ selon l'expression de Günther Anders.

C'est la tentative de faire bilan de ces effondrements qui est donc d'abord mise en avant. Dans cet "obsceno siglo" (91), il convient de faire état de ce qui est perdu, de nos objets mourants. Le poème "Tratamiento de residuos" (40) ne dit pas ce que laisserait entendre un tel titre. On s'attend sans doute à un discours moralisateur sur le bien trier ou le bien jeter. Mais le poème prend ouvertement le contre-pied de cette approche. Le poète, rudologue le temps d'un poème, étudie les déchets de nos modes de production et de consommation. Ici les rebuts et autres objets mourants sont : l'eau, l'air, la faune et la flore, le travail humain, l'histoire, l'art, la vie. Loin des fantasmes progressistes, ce court poème constitue une critique à peine voilée de la rhétorique machiniste et invite à reconsidérer la croyance d'une technique gage d'abondance et de bonheur.

Si Italo Calvino dit vrai quand il affirme que «L'acte de jeter est la première condition indispensable pour être, parce qu'on est ce qu'on ne jette pas ${ }^{29}$, alors il ne reste pas grand-chose à Homo detritus ${ }^{30}$. Une société qui considère la vie comme un déchet semble promise aux pires destinées.

\section{Relier les causes et les effets : rhétorique du « trop » et exponentielles}

Pour rendre sensible l'urgence de l'effondrement, le poète travaille à relier ce qui devait, pour les esprits empêchés, être impérativement délié. Et les images de l'épuisement du monde se disent peut-être d'abord dans le régime du « trop ». Il existe un mot pour dire le vouloir toujours plus : la pléonexie ${ }^{31}$. Comme le " pléonasme » est le mot en trop, l'excès de mot, la "pléonexie » est le désir de vouloir plus, plus que les autres en toute chose, plus que sa part, de posséder davantage, c'est la boulimie de l'avoir. Pour dire le régime pléonexique, l'écriture va jouer des effets de répétitions et de saturations parfois jusqu'à l'écœurement des mots :

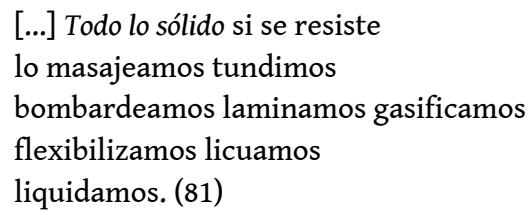

L'expression du trop se dit dans l'énumération, dans l'effet de liste et de recensement, dans le déploiement de l'arsenal d'actions à produire et à reproduire, dans le retour du même ad nauseam. Ces premières personnes du pluriel disent l'intensification des actions, la surenchère d'exécutions, le passage à la moulinette dans une mécanique de broyage. On retrouve là les maître-mots de la doxa néolibérale : mobilité, souplesse, flexibilité... 


\section{EL INQUILINO PREGUNTA}

En la casa donde vivo los restaurantes modernos

ofrecen aguas minerales de diez países diferentes

$\mathrm{y}$ vinos ecológicos, $\mathrm{y}$ en las tiendas new age

los ventiladores giran con suma lentitud

y puede fotocopiarse en papel reciclado.

Todas las ventanas dan al mismo horror.

Pero de dónde entonces

esa insensata aspiración inextinguible, necia,

de salvarnos, salvarnos. (93)

Ce choix en eaux minérales rappelle ces pages inondées de "trop" dans le petit opuscule de Jean-Louis Fournier sobrement intitulé Trop ${ }^{32}$ : trop de beurre, trop de yaourts, trop de brosses à dents... Le cerveau en mode « essorage $»^{33}$ n'est plus capable d'appréhender une telle profusion d'offre. L'idée du trop-plein est également présente dans ce poème des flux de démesure (117-118) :

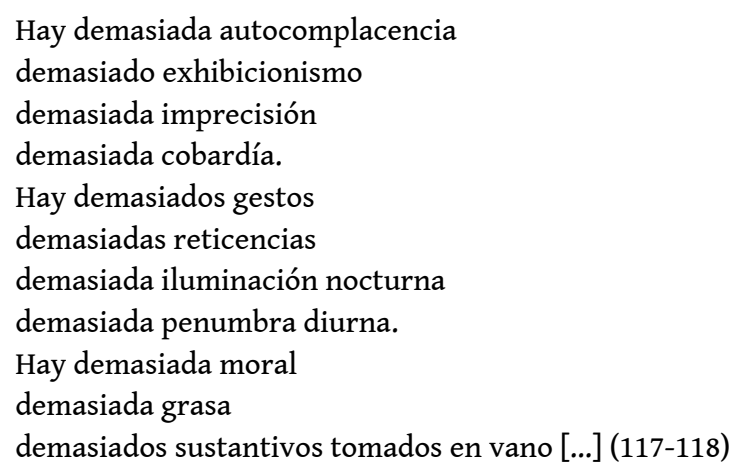

On atteint là le trop-plein et le point de saturation. Le poète décrit un monde dominé par le tourment de l'hubris et montre que les plaisirs et les conforts de court terme soutiennent une monumentale irrationalité de long terme. La mécanique des systèmes de répétitions atteint un nouveau palier avec la reprise d'une célèbre comptine pour enfants "Caballito blanco" :

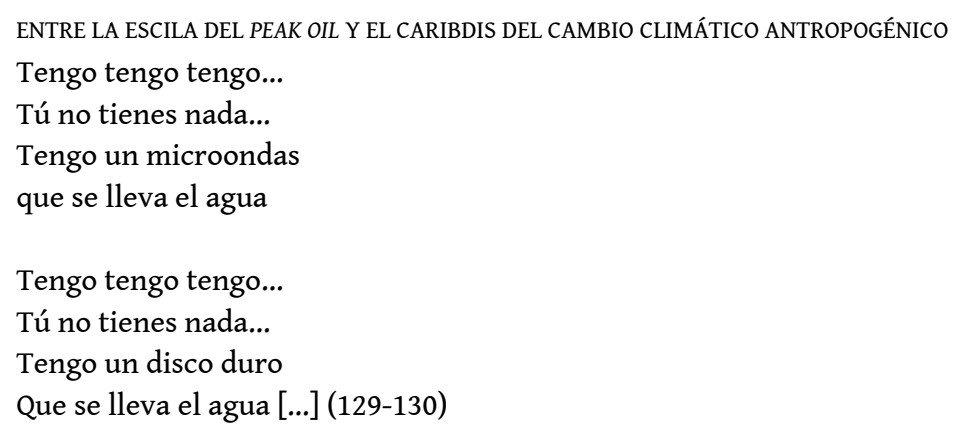

Sur huit couplets l'eau emporte ainsi le four à micro-ondes, le disque dur, le chéquier, la voiture, une cave, un pavillon, une piscine, trois amants, cent projets et un mensonge. La liste fournie est ici celle des possessions acquises au long d'une vie, les conquêtes matérielles et autres emplettes du tempérament domestique. Mais le poème nous dit que le bonheur n'est pas dans ces objets dont l'accumulation nous réjouit. L'énumération des biens de consommation dans «La complainte du progrès » de Boris Vian complèterait sans doute à merveille cette liste: aux côtés du micro-ondes, on trouverait « Mon frigidaire, mon armoire à cuillères / Mon évier en fer, et mon poêle à mazout / Mon cire-godasses, mon repasse-limaces ». Une force brûlante, l'obnubilation 
de l'avoir, invite à s'embarrasser de l'inutile. Mais tout passe, tel est le sens de ce poème qui dénonce la vanité de l'agitation de possession. En effet, les insouciances, les passions acquisitives, les regards détournés de l'essentiel sont interrogés et remis en question dans un choc d'images: d'un côté le monde de l'enfance dans la musicalité innocente du texte de la comptine. Et de l'autre, le titre qui mentionne les redoutables monstres de l'Odyssée, Charybde et Scylla ici revisités dans une lecture qui met en scène le choc pétrolier et le changement climatique.

Liquidation des stocks, tout doit disparaître... Le surplus, la société marchande et son monde sont à vendre dans un poème qui allie la description du trop-plein, des abus et leur dénonciation :

\section{LIQUIDACIÓN POR CAMBIO DE NEGOCIO}

De la emigración a Alemania con una maleta de cartón al turismo planetario, mientras apaleamos a los inmigrantes en casa, en una sola generación. Qué hemos dejado que hagan de nosotros...

El Caribe

el Mar Rojo

las islas del Pacífico

visite los sobrantes de selva tropical

los restos de tundra

los arrecifes de coral antes de que el desorden carbónico

acabe con los últimos

vuelos baratísimos

sexo baratísimo con niños y muchachas

exotismo- nada más que lo justo - igualmente asequible

descuentos especiales para funcionarios

relájese consumiendo su buena tajada del mundo

mastique a dos carrillos

visite los últimos paraísos

antes de que cierren

por cambio de negocio

tanta belleza tan barata

visite sus posesiones coloniales. (46-47)

“[...] relájese consumiendo su buena tajada del mundo", profitez de prix réduits sur les destinations bientôt disparues. Vente flash sur les Caraïbes, la Mer Rouge, les îles du Pacifique. Tourisme macabre et obscène. Le monde lui-même devient objet de consommation dans un poème qui, en poussant au maximum les curseurs de la dérision, ventriloque le discours publicitaire en employant ses codes discursifs ("visite", "acabe", "relájese", "mastique") et en mimétisant ses procédés de séduction (brièveté de la promotion, ventes privées...). La consommation du monde, c'est sa dégradation irrémédiable par un usage démesuré, abusif et inconséquent. Faisant disparaître le monde, l'homme disparaît lui-même. La dérive est suicidaire. Comment ne pas penser au châtiment d'Érysichthon, celui qui pour satisfaire son appétit sans fin finit par se manger lui-même. Dans le carpe diem inconséquent porté par le poème, le monde semble voué à l'autodestruction. Il est dévoré, digéré: "mastique a dos carrillos", c'est Érysichthon qui «agite en vain ses mâchoires » ${ }^{34}$. Avidité du monde, 
hubris impossible à apaiser : l'espèce humaine, autophage ${ }^{35}$, «nourrit son corps en le diminuant $»^{36}$.

Le monde se laisse aller à vau-l'eau, il court à sa perte dans un mouvement accéléré de fuite en avant. La question coloniale, celle de l'extinction des espèces et celle du tourisme sexuel se trouvent brassées dans un pêle-mêle des réalités insoutenables qui tiennent de niveaux de gravité et conséquence différents (délit, crime, abus, exploitation, déprédation...).

Cependant tous ne sont pas visés par les flèches de l'ironie railleuse du poème. La cible de cette publicité aux promesses engageantes est un segment somme toute précis de la population. Ce sont les privilégiés du "premier monde », ceux qu'Yves Citton qualifie d'« aristocrates planétaires » :

Avec notre eau courante et notre électricité sans coupures, avec nos passeports qui traversent les frontières comme du beurre, avec nos soixante marques de dentifrice, nos tomates en hiver, nos chaussures soldées et nos packs vacances tous frais payés sur une plage exotique, nous bénéficions d'un statut privilégié d'aristocrates planétaires qui n'est en rien moins inique que celui de la noblesse de $1788 .{ }^{37}$

La question de la responsabilité est elle aussi posée haut et clair dans les textes de Riechmann.

Un autre défi est aussi celui de représenter le schéma de l'exponentielle qui caractérise nos temps présents ${ }^{38}$. La difficulté est celle d'appréhender les spécificités d'un certain type de courbes: les grandes accélérations de croissance (de consommation et de production). La difficulté pour la parole poétique est d'être capable de représenter cette exponentielle en travaillant les tensions qui rendent laborieuse ces représentations mentales. En effet, montrer l'urgence demande à mettre en lumière le phénomène de croissance exponentielle. Un poème s'y risque par exemple en donnant une suite mathématique qui se définit par le calcul cumulé du double, une progression sur le mode de l'exponentielle :

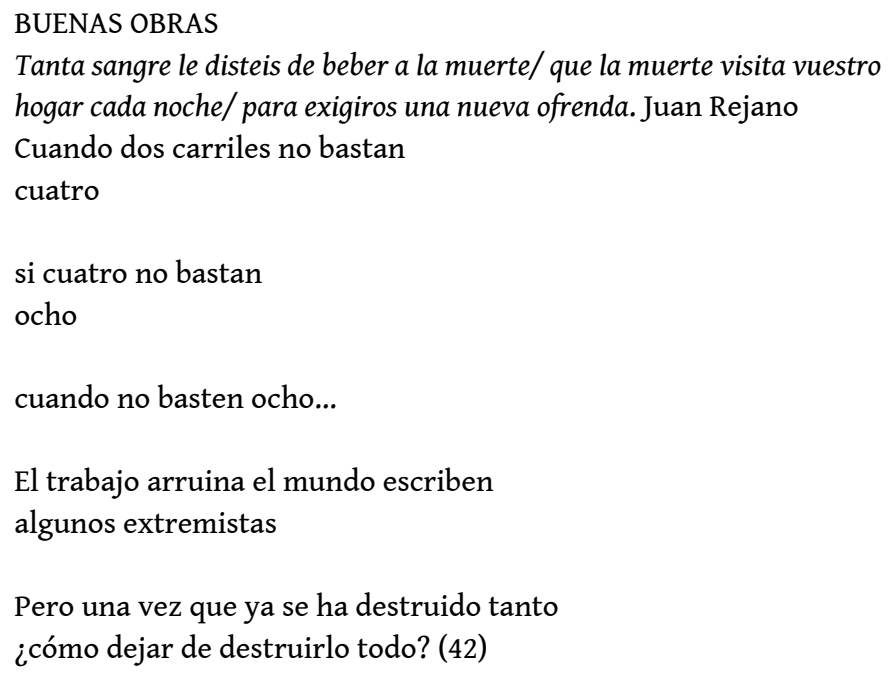

Le poète ânonne la suite mathématique au rythme d'une subordonnée temporelle, une subordonnée conditionnelle puis une nouvelle subordonnée temporelle. L'exhaustivité étant impossible, ce sont des points de suspension opportuns qui permettent de dérouler la séquence. L'exponentielle si parfaitement expliquée dans L'équation du nénuphar d'Albert Jacquard ${ }^{39}$, c'est une croissance à accélération constante, une fuite en avant qui, dans un monde fini, ne peut se terminer que dans l'insoutenable et dans 
l'intenable. Ici, une interrogation clôt le poème. Elle met en lumière l'absurdité qui empêche le retour en arrière. Les vaisseaux sont définitivement brûlés.

L'idée de " progrès » est également mise à mal dans les poèmes de Jorge Riechmann. Le progrès entendu comme «innovation » ou "modernité technique » et non le progrès compris comme force émancipatrice. Ce mot fétiche dit combien le péché d'orgueil régit nos mondes contemporains. Victor Hugo parlait du progrès qui « écrase » : «Sans cesse le progrès, roue au double engrenage, / Fait marcher quelque chose en écrasant quelqu'un. $\star^{40}$ Jorge Riechmann montre aussi les effets dévastateurs de cette course effrénée qui rabougrit l'humain, conduit à la tyrannie de la vitesse et accélère les ravages environnementaux. Il invite à déconstruire le culte en mettant en garde contre les notions frelatées de progrès: "[...] el progreso / del que te hablan envarados sacamuelas / con alfiler de corbata y estadísticas" (290), "Hay mucha estafa y engaño / en lo que llaman progreso" (126). Bien sûr, la question du progrès envahit abondamment le champ de la philosophie, de la politique et des sciences, mais c'est presque ici une simple question de bon sens qui est posée: sommes-nous prêts à accepter les limites ? Technologues et technophiles sont alors transpercés par les pointes de flèches ironiques : "[...] siguen produciendo y consumiendo como si tal cosa, tecnológicamente ubérrimos, cadavéricamente eufóricos" (218). Cette question a d'ailleurs pour corollaire celle de la vitesse et de l'accélération :

COMPETITIVIDAD (II)

Quienes van muy deprisa hacia ninguna parte

cierran el paso sin un sólo resquicio

a quienes queríamos llegar lentamente a un destino. (241)

\section{Mettre à nu : la parole poétique contre le blanchiment du langage}

En poésie, il est courant que les ripolinages lexicaux s'emmaillent significativement pour laisser place au dévoilement du réel. Celle de Riechmann s'occupe de montrer obstinément les détournements du langage et de dénoncer les fétichismes lexicaux. Il signale par exemple avec clarté les effets de blanchiment de langage qui ont pour corollaire de périlleuses contradictions :

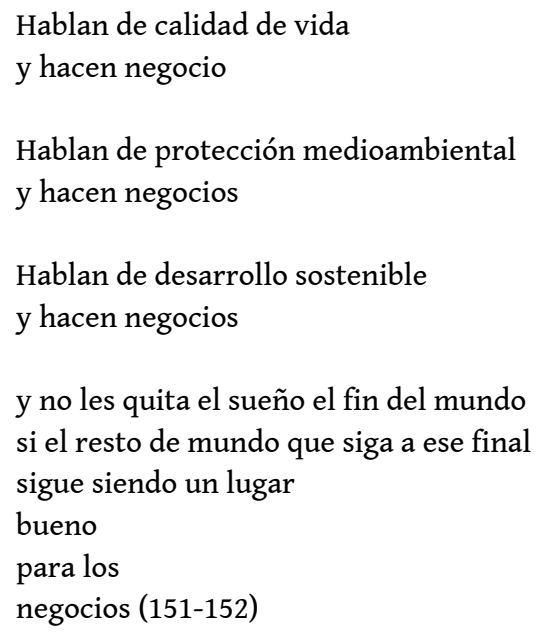

Dans l'analyse de la situation actuelle, le poème nous invite à considérer conjointement les hypothèses concurrentes de l'imbécilité et du cynisme. Plus aucune contradiction n'est aujourd'hui censurée. Ubu est roi : c'est le règne de l'absurde, du mensonge et de l'oxymore. Cette dernière figure est d'ailleurs l'outil de prédilection des justifications 
les plus irrationnelles. Bertrand Méheust voit dans l'usage de cette figure un symptôme qui ne trompe pas :

Plus l'on produit d'oxymores et plus les gens sont désorientés et inaptes à penser. Utilisés à doses massives, ils rendent fou. Plus la crise s'aggrave, plus le réchauffement climatique nous menace et plus nous assistons à la production et à l'usage cynique, sans précédent dans la démocratie française, d'oxymores à grande échelle. ${ }^{41}$

Le poème "Balance provisional de la guerra contra Irak" (265-266) met en scène les mots de la guerre et la guerre des mots. Il est ici question de contre-attaque sémantique : la parole poétique devient une arme de destruction des trahisons du réel par un langage corrompu et pernicieux. C'est la figure de Socrate qui préside au travail de dévoilement du réel :

[...] Sócrates dijo una vez que el uso perverso del lenguaje

inocula el mal en el alma

Nuestras palabras

¿quién las descontaminará?

nuestros pensamientos

¿cuándo serán liberados? (265-266)

41 La poésie est le lieu de l'offensive. Dans ce poème, ce sont les faits de violence et de barbarie qui sont replacés au cœur des mots "democracia / libertad / civilización". Que peut la poésie ? Démonter à la clé à molette et au tournevis les formules et les motsemblèmes. Montrer que les mots ne sont pas neutres. La poésie, qui par nature rompt avec le langage ordinaire, possède cette force de frappe qui marque les esprits. Cette bataille sémantique est en soi une action politique. On est tenté ici de convoquer les écrits politiques de Brecht qui appelaient au « rétablissement de la vérité ${ }^{42}$. Au sujet de cette injonction à mener certaines guerres, il disait déjà l'impérieuse nécessité de l'analyse des discours: "Lorsqu'il [le penseur] entend dire que les guerres sont nécessaires, il ajoutera [...] dans quelles circonstances elles le sont, et pour qui $»^{43}$.

Le rôle du poète est aussi sans doute de faire une halte sur les mots, de mettre à nu. Un poème alerte ainsi sur les postures et les impostures de formulations qui rendent la pensée captive. Jorge Riechmann travaille alors dans son écriture à déminer les pièges lexicaux pour remettre le monde d'équerre :

CURIOSAMENTE

SUELE llamarse realismo político

a la mirada ciega

hacia todos los aspectos de la realidad

que no sean las relaciones de poder

entendido más bien como una fuerza bruta:

una mirada muy poco realista. (150)

Le réalisme, nous dit le poème, n'est donc sans doute pas là où on voudrait bien le voir.

\section{Hybridations : du marketing et de l'économie politique en poésie}

On le sait - et Roland Barthes l'a clairement expliqué dans ses analyses de sémiologie publicitaire - le marketing et la réclame ont fait main basse sur la poésie : 
[...] le « bon » message publicitaire est celui qui condense en lui la rhétorique la plus riche et atteint avec précision (souvent d'un seul mot) les grands thèmes oniriques de l'humanité, opérant ainsi cette grande libération des images (ou par les images) qui définit la poésie elle-même. Autrement dit, les critères du langage publicitaire sont ceux-là même de la poésie [...]. ${ }^{44}$ "Razones para la acción" (178) renvoie sans doute immédiatement au collectif créé autour de Bourdieu pendant les mouvements de grève de l'hiver 1995, "Raisons d'agir ». La première strophe met en avant, dans une anesthésiante litanie d'épithètes, les nombreux obstacles qui enlisent la mise en action. Cependant, dans le tercet et le quatrain qui suivent cette longue strophe, la réaction advient malgré tout. Celle-là même qui refuse la capitulation pressentie et attendue dans les seize premiers vers. Tout ce qui était entrave devient alors raison d'agir. Envers et contre tout, en dépit de tout, encore et toujours - pour reprendre là le rythme obsessionnel de la litanie - la parole poétique ne consent pas au renoncement. Les conjonctions adversatives en position initiale de strophe encouragent à la reprise du flambeau: "Pero no soy un vegetal indiferente, sino un ser humano", "Pero no soy un hombre sino un bosque". L'épanorthose, reprise corrective qui vise la justesse, est la réponse radicale à l'anesthésie: elle permet de dire avec précision la pensée vivante, dynamique et plurielle. 
ême phénomène d'obstination est présent dans le poème "Desear" (116). Des deux côtés de la pause strophique, ce sont deux mondes opposés qui se font face :

Desear

que siga existiendo el mundo para que siga existiendo

toda la belleza del mundo

es una ingenuidad

a la que no renunciamos.

(In

genuo significa

etimológicamente

nacido libre) (116-117) vers posent une déclaration qui invite à rendre les armes. Découragement, épuisement, inutilité : c'est le drapeau blanc de la reddition qui semble ondoyer dans ces quatre vers dont la disposition engage à voir le déploiement sur la page. Mais l'enjambement inattendu qui projette et enlace un cinquième vers isolé, "a la que no renunciamos", contrarie le sens si promptement admis d'abord par le lecteur. La suspension strophique et métrique offre un retournement efficace et dit combien l'engagement est solide mais surtout constant. L'emploi d'une première personne du pluriel vient renforcer la puissance du vers qui affirme la persévérance du combat. Ce sujet collectif a bien sûr des enjeux éthiques, philosophiques et politiques : c'est le nous du commun, tous noués et interdépendants ${ }^{45}$. texte $^{46}$ - et de l'utopie. Plus avant, dans le paratexte, une sentence du dessinateur $E l$ Roto met aussi à nu l'illusion nominale des étiquettes et les fonctions idéologiques des cases qui offrent du "prêt-à-penser »: “¡Qué manía de llamar utópico a lo que no permiten!" (117).

51 Le poème "El fin de la esclavitud" (150) montre aussi comment les utopies d'hier sont devenues les réalités d'aujourd'hui. Voilà des textes qui appellent à construire un monde où l'action serait sœur du rêve. L'esprit de l'utopie est puissamment présent dans ces textes: utopies réelles, utopies concrètes, on parlerait aujourd'hui aussi de "possitopies $»^{47}$. Pour déployer de nouveaux horizons, de nouveaux imaginaires, pour construire des « demains désirés », la bataille se mène dans les têtes et par la réflexion :

La lucha

no se decide en las calles

sino en los callejones

de la conciencia. (119)

On retrouve ici la même technique de la pause suspensive à l'enjambement. Le temps de la suspension est justement l'invite à la réflexion, à la prise de recul, au raisonnement. Alors, envers et contre-tout, il faut tenter d'imaginer la " perspective des délices ». Une maxime propose de conjurer l'impossible : "Improbable la orquídea, pero no imposible" (182). La raison du combat : prendre le risque d'essayer.

53 C'est le rythme de la nature, la sagesse végétale et minérale qui montre la voie à suivre. Le poème "Voluntarismo vegetal" (181) pose la volonté, l'agir comme une prise de risque. Ni renonçant, ni résigné, le poète se laisse inspirer par le volontarisme de la nature. Ce sont les amandiers, genêts, prunelliers, abricotiers qui montrent cette voie. 
En effet, leur floraison précoce a pour corrélat une prise de risque, une mise en danger, mais le pari est passionnel, audacieux, courageux: "vuestra floración temprana [...] supone un arriesgado acto de fe [...]. Hay algo de ese voluntarismo irremediable en todas las búsquedas que atina a emprender el ser humano" (181). C'est ici un poème en prose, poème « insoumis[sion] au vers » comme le dit Jean-Michel Espitallier ${ }^{48}$, qui dit la prise de risque et l'audace du végétal qui envahit la page.

\section{Un contrat jubilatoire avec le monde}

54 L'écriture de Riechmann porte une vitalité singulière. Elle laisse la place à ce qu'il appelle ses "privilèges» ("Privilegios", 200) et ses "plaisirs» (“Placeres", 199). Le plaisir n'est pas tant l'objectif que le moteur même. Il y a quelque chose de déroutant dans ces poèmes jubilatoires qui cohabitent avec les poèmes de l'effondrement et les poèmes de la barbarie. La douceur déconcerte d'abord. Puis l'on comprend que la célébration du monde est première malgré tout. Le poème "Yo celebro" (48) pose ce qui ressemble au paradoxe du chant poétique sur les ruines du monde :

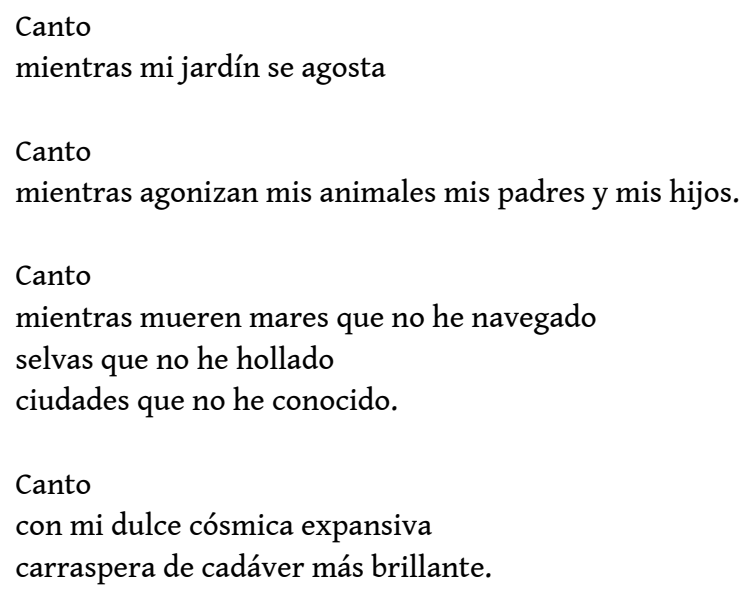

La poésie de l'effondrement est comme une quinte de toux incoercible, qui écorche mais qui doit se livrer. L'anaphore dit ici l'entêtement à voir et à dire. Les regards sur le monde se multiplient: le poète est tour à tour astrophysicien, spéléologue, entomologiste, botaniste...

\section{L'entrée en résonance}

Le dernier ouvrage du sociologue et philosophe allemand Hartmut Rosa, Résonance. Une sociologie de la relation au monde, pourrait constituer un point d'appui précieux pour comprendre les tentatives de réponses à l'urgence dans (et par) la parole poétique. Penseur du dérèglement entre les hommes et le monde, Hartmut Rosa avait analysé dans son ouvrage Aliénation et accélération ${ }^{49}$ des phénomènes de désynchronisation et avait fait état des injonctions à la vitesse continue dans la période présente de modernité tardive. Constat et bilan qui semblent partagés par Jorge Riechmann.

La réponse - une des réponses -, est de s'initier à « habiter le monde » et se l'approprier grâce à une relation de résonance. Celle-ci commence sans doute (mais pas nécessairement) par une première étape de décélération. Se réapproprier le temps, apprendre la lenteur, se libérer de la force «totalitaire » de la vitesse, voilà le premier apprentissage : 
Déjate recorrer por los caminos

Ganarás tanto tiempo:

el tiempo de la vida (240)

COMPETITIVIDAD (II)

Quienes van muy deprisa hacia ninguna parte

cierran el paso sin un sólo resquicio

a quienes queríamos llegar lentamente a un destino. (241)

¿Se comerá lo rápido a lo lento?

Está por ver. Podemos mientras tanto

Masticar muy despacio; (228)

Le pas de côté permet de prendre conscience que «le temps est hors de ses gonds » selon la formule shakespearienne ${ }^{50}$. La poésie est peut-être alors l'embrasure qui permet ce petit pas de côté. Elle enseigne à vibrer, elle initie à l'art de la résonance, elle est un exercice de connaissance pour saisir le monde. Ce sont ainsi les formes brèves qui éveilleront aux frissons du réel. La greguería d'abord qui, parce qu'elle est de l'ordre de l'évidence poétique, joue d'une observation élémentaire du monde pour comprendre les liens :

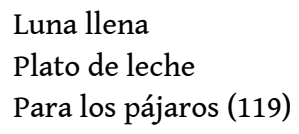

La greguería est ici une approche intuitive du monde. Elle est le signe d'une relation de jouissance au monde. La greguería enseigne ce qui advient de façon minimale, ce qui est vibrant et palpitant. Le format du haïku permet sans doute cela aussi. Ils sont d'ailleurs nombreux dans l'anthologie. C'est là une connaissance par les flèches et non par les gouffres.

Necesitaba

todo un año de viento

contra la cara (145)

La voix de la poésie est celle qui permet alors une sensibilité nouvelle au monde, un rapport engagé au monde. Il y a aussi dans l'écriture du fragment, quelque chose de l'ordre du plaisir des débuts. Autant de fragments, autant de commencements. C'est Barthes qui avait corrélé l'écriture du fragment à la notion de plaisir: "Autant de fragments, autant de débuts, autant de plaisir $»^{51}$. Le plaisir aigu de commencer un texte renvoie peut-être aussi à une forme d'espoir en un nouveau commencement possible. Un poème invite de façon convaincante à une autre sensibilité au monde :

He vivido en la superficie de las cosas.

Mas viví también por fortuna

en las palabras. Ellas iban

incorporándome a la lentitud

penetrando las estaciones de mi piel

dilatando la malla amarga de los días

rastreando el frío y el calor en los seres

zambulléndose en el amor hasta salir al tedio

en el tedio hasta dar en la esperanza

en la esperanza hasta emerger en el asombro

sin yo quererlo o porque lo quería.

Las palabras 
maravillosamente

incapaces de compromiso.

No soy un juglar de la descomposición. Acaso

amo sobre todas las cosas

el lugar del canto del pinzón

la aérea ebriedad de las mimosas

y el minuto con memoria del beso de los amantes.

Pero hay que ir hasta el fondo

correr el riesgo

de abrasarse en la resistencia de las cosas

- para sacar acaso la cabeza

al otro lado del espejo

o en el frescor de un nuevo meridiano. (99-100)

61 C'est un poème qui donne une clé de lecture à ces différentes « façons de répondre aux coups portés » et à l'urgence du moment. La question de la sensibilité au monde est au cœur de ce texte qui est d'un éclat et d'une puissance décisifs. Il y est question de l'importance de la relation corporelle au monde. La peau, cette première surface de résonance et "organe littéralement respirant et répondant $~_{52}^{2}$ explique Harmut Rosa, invite à cette exploration, ici: dilater, traquer, plonger, s'immerger dans les profondeurs, aimer. Le média qui permet cette compréhension et cet accès à la résonance est identifié dans ce texte : c'est le matériau poétique lui-même, ce sont les mots.

Les effets de synesthésie sont robustes et le poème possède aussi un fort pouvoir de mobilisation des imaginaires. L'expression "las estaciones de mi piel" dit ce rejet du temps compté, comptabilisé, imposé, le temps mécanique du tic-tac régulier de l'horloge. Le temps de la peau est en effet celui de la nature avec ses fluctuations, ses approximations, le rythme de l'improvisation. Non pas les années, les mois, les jours mais la saison des fruits, la saison des orages, la saison du retour de la sève...

L'apprentissage et l'accès à la lenteur ("incorporándome a la lentitud") font écho aux concepts développés par Hartmut Rosa de la «pénurie du temps» dans des époques marquées par l'accélération technique et l'accélération sociale. La lenteur dit Hartmut Rosa, c'est "la richesse du temps " ${ }^{53}$. La synesthésie présente dans l'expression "la malla amarga de los días" vient dire également le corset amer d'un temps qui peut se compter aujourd'hui en micro secondes. L'expression nous rappelle que notre rapport au temps se résume bien souvent à un seul sentiment : celui d'en manquer.

Dans un moment où le présent pèse et les lendemains inquiètent, l'écriture de Jorge Riechmann ne s'inscrit cependant pas dans une démarche crépusculaire. Il invite d'ailleurs à se délester du pessimisme et du défaitisme : "Dejemos el pesimismo para tiempos mejores" dit un graffiti repris dans le recueil (214). Son travail «a pour nom combat $\aleph^{54}$ - pour paraphraser ici l'expression de Günter Anders - et il s'attache à rendre sensible les effets de simultanéité : ce sont les locutions conjonctives « pendant que ", « au moment où », « tandis que », « dans le même temps que » qui vont porter la question de l'urgence et la nécessité d'agir ${ }^{55}$. L'urgence est donc celle de reconnecter ces deux temporalités non sécantes. L'urgence est de rendre sensible cette réalité à un lecteur oublieux qui «nuit et jour à tout venant » ne songe qu'à chanter... L'urgence n'aura sans doute que faire des délibérations interminables et complexes.

Pour nous arracher à l'indifférence, Riechmann met d'abord en pratique le commandement de Günther Anders : «Inquiète ton voisin, comme toi-même » ${ }^{56}$. En 
effet, dans un premier geste d'écriture, il nous impose un deuil redoutable et porte ainsi le fer et le feu dans les plaies de notre modernité dévastatrice. Mais dans un deuxième temps, invitant à se prémunir du pire, il explore les vertiges d'un monde désiré, il propose de faire monde différemment : "Cualquier lugar puede ser el paraíso, también éste" (184). Ce désespoir souriant et cet enthousiasme déraisonnable nous invitent à imaginer le poète en "Sisyphe heureux " $^{57}$.

Mais quel est l'espace d'action pour l'œuvre créatrice ${ }^{58}$, quel est rôle de la poésie ? “[...] no hay poema que deje al mundo intacto" affirme Riechmann. Il est alors sans doute une tâche offerte aux artistes, non pas changer le monde mais peut-être plus que jamais et comme le disait Camus "empêcher que le monde ne se défasse " ${ }^{59}$. La poésie pour rendre sensible, pour faire éprouver, pour affecter les corps, pour appréhender, pour s'emparer : “[...] los casos peores de desnutrición / se dan en gentes que no comen poesía" (282).

\section{NOTES}

1. Les citations qui apparaissent entre parenthèses dans cet article sont les références des pages de l'ouvrage soumis à analyse: Jorge Riechmann, Con los ojos abiertos (ecopoemas 1985-2006), Tenerife, Ediciones Baile del sol, 2007.

2. Jorge Riechmann, El Corte bajo la piel in Futuralgia, Madrid, Calambur, 2011, p. 522.

3. Nicanor Parra, Obra gruesa, Santiago de Chile, Editorial Andrés Bello, 1983, p. 157.

4. Ibid.

5. En cela, le poète rejoint sans doute le champ de recherche transdisciplinaire qu'est la collapsologie. Cette étiquette proposée par Pablo Servigne et Raphaël Stevens serait presque une boutade si le sujet n'était pas aussi sérieux : les collapsologues étant ceux qui s'intéressent au désastre qui vient. Pablo Servigne, Raphaël Stevens, Comment tout peut s'effondrer. Petit manuel de collapsologie à l'usage des générations présentes, Paris, Éditions du Seuil, 2015.

6. Maurice Blanchot, L'Écriture du désastre, Paris, Gallimard, 1980.

7. Yves Citton, Renverser l'insoutenable, Paris, Seuil, 2012, p. 17-51.

8. Déborah Danowski, Eduardo Viveiros de Castro, «L'Arrêt du monde » in Emilie Hache (ed.), De l'univers clos au monde infini, Paris, Éditions Dehors, 2014.

9. Anthony D. Barnosky (et al.), "Approaching a state shift in Earth's biosphere”, Nature, vol. 486, p. 52-58, 07 juin 2012, https://www.nature.com/articles/nature11018 [consultation juillet 2018].

10. Bruno Latour, Où atterrir ? Comment s'orienter en politique, Paris, La Découverte, 2017, p. 15.

11. Ibid., p. 37.

12. Pablo Servigne, Raphaël Stevens, Comment tout peut s'effondrer. Petit manuel de collapsologie à l'usage des générations présentes, op. cit, p. 229.

13. Ibid., p. 228. 
14. « Nous sauver de la catastrophe, c'est parvenir à empuissanter - à temps - les idées vraies impuissantes de la science climatique, c'est-à-dire à nous donner les figurations vivaces des choses à venir pour que, enfin associées à des images qui (dit-on) "frappent les esprits" (en fait elles sont des affections de notre corps), les vérités de la science climatique cessent de n'être que des idées idéelles. Et acquièrent le pouvoir de nous affecter ", Frédéric Lordon, Les affects de la politique, Paris, Seuil, 2016, p. 59-60.

15. Jean-Pierre Dupuy, Pour un catastrophisme éclairé. Quand l'impossible est certain, Paris, Seuil, 2002, p. 175.

16. René Char, Fureur et mystère, Paris, Gallimard, 1962, p. 126.

17. Hans Jonas, Le Principe responsabilité, Paris, Flammarion, 1995, p. 233.

18. François Cusset, Le Déchaînement du monde. Logique nouvelle de la violence, Paris, La découverte, 2018 , p. 10.

19. François Cusset, Le Déchaînement du monde. Logique nouvelle de la violence, op. cit., p. 6.

20. Serge Latouche, Le Pari de la décroissance, Paris, Fayard, 2006, p. 41.

21. «Le problème est que nous ne croyons pas. Nous ne croyons pas ce que nous savons. Le défi qui est lancé à la prudence n'est pas le manque de connaissance sur l'inscription de la catastrophe dans l'avenir, mais le fait que cette inscription n'est pas crédible. [...] Nous tenons la catastrophe pour impossible dans le même temps où les données dont nous disposons nous la font tenir pour vraisemblable et même certaine ou quasi-certaine ", Jean-Pierre Dupuy, Pour un catastrophisme éclairé, Quand l'impossible est certain, op. cit., p. 142.

22. Il n'apparaît dans l'ouvrage que sous la plume du poète et critique Niall Binns qui signe l'épilogue (311-312), sous celle du professeur d'économie Óscar Carpintero en charge de l'introduction (10) et enfin sous la plume du sociologue Ernest García dans une épigraphe qui ouvre le recueil (24).

23. On le retrouve aussi dans l'épilogue de Niall Binns : trois occurrences (312 et 317).

24. Et ce sont précisément ces premiers vers qui sont cités et commentés dans l'introduction et l'épilogue (15 et 321).

25. Günther Anders, Endzeit und Zeitenende. Gedanken über die atomare Situation, Munich, C.H. Beck, 1972.

26. "Une humanité qui traite le monde comme "un monde bon à jeter" se traite elle-même comme "une humanité bonne à jeter" ", Günther Anders, L'obsolescence de l'Homme (sur la destruction de la vie à l'époque de la troisième révolution industrielle), tome II, Paris, Fario, 2011, p. 44. 27. Günther Anders, Hiroshima est partout, Paris, Seuil, 2008.

28. "On nous a traité de "semeurs de panique". C'est bien ce que nous cherchons à être », Günther Anders, Et si je suis désespéré, que voulez-vous que j'y fasse ? Entretiens avec Mathias Greffrath, Paris, Éd. Allia, 2001.

29. «Il buttar via è la prima condizione indispensabile per essere, perché si è ciò che non si butta via», Italo Calvino, La strada di San Giovanni, Milano, Mondadori, 1990, p. 97.

30. Baptiste Monsaingeon, Homo detritus. Critique de la société du déchet, Paris, Seuil, 2017.

31. Dany-Robert Dufour, Pléonexie [dict. : «Vouloir posséder toujours plus»], Lormont, Le bord de l'eau, 2015.

32. Jean-Louis Fournier, Trop, Paris, La Différence, 2014, p. 125.

33. «Mon cerveau est sur lavage. Mon cerveau est sur essorage» (ibid., p. 12).

34. Ovide, Les Métamorphoses, Paris, Gallimard, p. 284.

35. Anselm Jappe, La société autophage, Capitalisme, démesure et autodestruction, Paris, La Découverte, 2017.

36. «Mais quand l'excès de la souffrance, ayant consumé tout ce qui lui servait de matière, donna une pâture nouvelle à son horrible maladie, Erysichthon se mit à déchirer lui-même ses propres 
membres à coups de dents; l'infortuné nourrit son corps en le diminuant» (Ovide, Les Métamorphoses, op. cit., p. 286).

37. Yves Citton, Renverser l'insoutenable, op. cit., p. 31.

38. Voir à cet égard le «Tableau de bord de l'anthropocène » in Pablo Servigne, Raphaël Stevens, Comment tout peut s'effondrer. Petit manuel de collapsologie à l'usage des générations présentes, op. cit., p. 34 .

39. Albert Jacquard, L'équation du nénuphar : les plaisirs de la science, Paris, Calmann-Lévy, 1998.

40. Victor Hugo, Les contemplations, Paris, Flammarion, 1995, p. 336.

41. Bertrand Méheust, La Politique de l'oxymore, Paris, La Découverte, 2009 (quatrième de couverture).

42. Bertolt Brecht, «Le rétablissement de la vérité » in Écrits sur la politique et la société, Paris, L'Arche, 1971.

43. Cité dans Bertolt Brecht 1, Cahier de L'Herne, dirigé par Bernard Dort et Jean-François Peyret, Paris, Éditions de L'Herne, 1979 p. 209.

44. Roland Barthes, "Le langage publicitaire, rêve et poésie ", Les Cahiers de la publicité, 1963, vol. 7, $\mathrm{n}^{\circ} 1$, p. 92-96.

45. La galerie d'art contemporain « Angels Barcelona » a accueilli une exposition ayant pour titre un extrait de ce poème, “Toda la belleza del mundo", qui mettait au cœur de la réflexion les concepts de " démocratie et citoyenneté universelle, économie politique dans les corps du capitalisme », http://angelsbarcelona.com/en/program/toda-labelleza-del-mundo/214 [consultation, juin 2019].

46. Le Littré rappelle aussi que l'ingénuité n'est pas la naïveté : « INGÉNUITÉ, NAÏVETÉ. Ces deux mots désignent le naturel et la simplicité; mais l'étymologie y met une nuance : l'ingénuité est ce qui est propre à une personne libre; la naïveté exprime seulement ce qui est natif. De là vient qu'ingénuité est toujours un éloge, tandis que naïveté touche quelquefois à une simplicité trop grande, à une sorte de niaiserie. » https://www.littre.org/definition/ing\%C3\%A9nuit\%C3\%A9 [consultation juin 2019] (nous soulignons).

47. Pablo Servigne, Raphaël Stevens, Gautier Chapelle, Une autre fin du monde est possible, Paris, Seuil, 2018, p. 173.

48. Jean-Michel Espitallier, Caisse à outils: un panorama de la poésie française aujourd'hui, Paris, Pocket, 2006, p. 164.

49. Hartmut Rosa, Aliénation et accélération, Vers une théorie critique de la modernité tardive, Paris, La Découverte, 2014.

50. Dans la traduction d'Yves Bonnefoy : William Shakespeare, Hamlet, Paris, Gallimard, 1978.

51. Roland Barthes, Roland Barthes par Roland Barthes, Paris, Seuil, 1975, p. 98.

52. Hartmut Rosa, Résonance. Une sociologie de la relation au monde, Paris, La découverte, 2018, p. 62.

53. Ibid., p. 43.

54. "Les expressions de "menace de l'humanité par elle-même" ou de "suicide de l'humanité" sont fausses. Nous devons renoncer au moindre espoir qui reposerait sur ces expressions. Le temps de la fin dans lequel nous vivons, pour ne rien dire de la fin des temps, contient deux sortes d'hommes : celle des coupables et celle des victimes. Nous devons tenir compte de cette dualité dans notre réaction : notre travail a pour 
nom "combat" ", Günther Anders, Le Temps de la fin, Paris, Éditions de l'Herne, 2007, p. 33.

55. "Se habla de música / de fútbol / del colegio de los niños // mientras nuestro consumo energético arrasa los cinco continentes" (257); "Canto mientras mi jardín se agosta" (48); "mientras se funden los hielos del planeta" (147); "Mientras la casa se quema" (49), "nos estamos quedando / quietecitos // mientras las cruces // donde agonizan / ahora / nuestros hermanos incontables" (89).

56. Günther Anders, Et si je suis désespéré, que voulez-vous que j'y fasse? Entretiens avec Mathias Greffrath, op. cit.

57. Albert Camus, Le mythe de Sisyphe, in Cuvres, Paris, Quarto Gallimard, 2013, p. 328.

58. L'espace pour l'œuvre créatrice est le vœu formulé par Yves Citton: «Entre l'Action (révolutionnaire) et les pressions (à vocation pacifiste), il convient peut-être de dégager l'espace d'un troisième domaine, qui serait celui de l'œuvre créatrice», Yves Citton, Renverser l'insoutenable, op. cit., p. 75.

59. "Chaque génération, sans doute, se croit vouée à refaire le monde. La mienne sait pourtant qu'elle ne le refera pas. Mais sa tâche est peut-être plus grande. Elle consiste à empêcher que le monde ne se défasse ", Albert Camus, Discours de Suède, in CEuvres, op. cit., p. 82.

\section{RÉSUMÉS}

L'œuvre du poète espagnol Jorge Riechmann (Madrid, 1962) nous rappelle que nous sommes aujourd'hui impérieusement requis de penser l'urgence du "Nouveau Régime Climatique» (Bruno Latour). Dans ses recueils, Riechmann semble nous dire que le statu quo et l'anosognosie ont fait leur temps. Son écriture se fraye une voie entre les rochers des certitudes et des aveuglements qui régissent notre arrogant $\mathrm{XXI}^{\mathrm{e}}$ siècle. Face aux défis sociaux-écologiques et aux inouïs de violence qui assaillent nos mondes contemporains, le poète fait sa part : «La poesía y el arte no pueden casi nada : pero ese casi nada es esencial no dejar de intentarlo " (Tuits para el siglo de la Gran Prueba). Le recueil Con los ojos abiertos (ecopoemas 1985-2006), objet d'analyse dans cet article, s'inscrit dans cette urgence du moment: une urgence tout à la fois politique, sociale et écologique. On interrogera ici la poétique de l'effondrement: quelles stratégies sont mises en place pour rendre compte de ce nouveau régime climatique? Quels mécanismes entrent en jeu pour faire prendre conscience de l'urgence? Quel est le rôle de la poésie pour rendre sensible et «donner des figurations vivaces » de la dégradation des environnements?

La obra del poeta español Jorge Riechmann (Madrid, 1962) impone considerar y enfrentar con lucidez la cuestión imperiosa del "Nuevo Régimen Climático" (Bruno Latour). Ante la urgencia ecológica los poemarios de Riechmann exigen una toma de conciencia. Su escritura se abre camino entre las rocas de las certezas y la ceguera que gobiernan nuestro arrogante siglo XXI. Ante los retos socio ecológicos y las nuevas formas de violencia que asolan nuestro mundo contemporáneo, el poeta "cumple con su parte": "La poesía y el arte no pueden casi nada: pero ese casi nada es esencial no dejar de intentarlo" (Tuits para el siglo de la Gran Prueba). El poemario Con los ojos abiertos (ecopoemas 1985-2006), en el que se enfoca el análisis de este artículo, se inscribe en esta urgencia del momento: una urgencia a la vez política, social y ecológica. Se propondrán algunos hitos y pautas para sondear esta "poética del colapso": ¿de qué estrategias se vale el 
poeta para dar cuenta de este nuevo régimen climático? ¿Qué mecanismos son empleados para crear conciencia sobre la urgencia del momento presente? ¿Cuál es el papel de la poesía para "dar figuraciones vivas" de nuestras vulnerabilidades?

INDEX

Mots-clés : urgence climatique, nouvelles formes de violence, écriture de l'engagement, écopoétique, Riechmann (Jorge)

Palabras claves : urgencia climática, nuevas formas de violencia, escritura del compromiso, ecopoética, Riechmann (Jorge)

\section{AUTEUR}

JUDITE RODRIGUES

Université de Bourgogne Franche-Comté, EA 4182 